\title{
Assessment of Acute and Chronic Pharmacological Effects on Energy Expenditure and Macronutrient Oxidation in Humans: Responses to Ephedrine
}

\author{
Antonella Napolitano, ${ }^{1}$ Peter R. Murgatroyd, ${ }^{2}$ Nick Finer, ${ }^{2}$ Elizabeth K. Hussey, ${ }^{3}$ \\ Robert Dobbins, ${ }^{3}$ Steve O'Rahilly, ${ }^{2}$ and Derek J. R. Nunez ${ }^{3}$ \\ ${ }^{1}$ Clinical Unit in Cambridge, GlaxoSmithKline, ACCI, Cambridge University Hospitals NHS Foundation Trust, Hills Road, \\ Cambridge CB2 2QQ, UK \\ ${ }^{2}$ Wellcome Trust Clinical Research Facility, Cambridge University Hospitals NHS Foundation Trust, Cambridge CB2 2QQ, UK \\ ${ }^{3}$ Metabolic Pathways Center of Excellence for Drug Discovery, GlaxoSmithKline Research Triangle Park, NC 27709, USA
}

Correspondence should be addressed to Antonella Napolitano, antonella.2.napolitano@gsk.com

Received 17 May 2010; Accepted 6 July 2010

Academic Editor: L. Van Gaal

Copyright (C) 2011 Antonella Napolitano et al. This is an open access article distributed under the Creative Commons Attribution License, which permits unrestricted use, distribution, and reproduction in any medium, provided the original work is properly cited.

Evidence of active brown adipose tissue in human adults suggests that this may become a pharmacological target to induce negative energy balance. We have explored whole-body indirect calorimetry to detect the metabolic effects of thermogenic drugs through administration of ephedrine hydrochloride and have assessed ephedrine's merits as a comparator compound in the evaluation of novel thermogenic agents. Volunteers randomly given ephedrine hydrochloride $15 \mathrm{mg}$ QID $(n=8)$ or placebo $(n=6)$ were studied at baseline and after 1-2 and 14-15 days of treatment. We demonstrate that overnight or 23-hour, 2\% energy expenditure (EE) and 5\% fat (FO) or $\mathrm{CHO}$ oxidation effects are detectable both acutely and over 14 days. Compared to placebo, ephedrine increased EE and FO rates overnight (EE $63 \mathrm{~kJ}$ day 2, EE $105 \mathrm{~kJ}$, FO $190 \mathrm{~kJ}$, day 14), but not over 23 h. We conclude that modest energy expenditure and fat oxidation responses to pharmacological interventions can be confidently detected by calorimetry in small groups. Ephedrine should provide reliable data against which to compare novel thermogenic compounds.

\section{Introduction}

Obesity usually develops gradually as a result of a modest but sustained energy surplus. A continuing positive energy imbalance of just $1 \%$ predicts a body mass increase of around $1 \mathrm{~kg}$ per year. Clinical management of obesity presents a particular challenge because lifestyle interventions are poorly effective [1-3] and there are few drug targets that have yielded outstanding clinical efficacy. Theoretically, such targets could be in systems regulating energy intake or energy expenditure, although it is clear that these are closely integrated within the central nervous system (CNS).

Most pharmacological approaches to obesity management in humans have focussed on reducing energy intake. Even when pharmacological interventions have been postulated to increase energy expenditure, it seems that much of the effect on body weight was mediated through decreased energy intake [4]. However, it is interesting that the ephedrine-caffeine combination [4] and, more recently, tesofensine [5], a triple central monoamine uptake inhibitor, which have been shown to produce weight loss, do have measurable effects on energy expenditure, though again most of the weight loss observed appears to be attributable to effects on energy intake [6]. This demonstrates that mechanistic specificity is hard to achieve. This is exemplified by sibutramine, a drug that predominantly enhances satiety, but which, in some studies, increases energy expenditure acutely and reduces the fall in resting energy expenditure seen during weight loss [7-9], although in others it was without measurable effect $[9,10]$. This contrasts with the substantial energy expenditure effects of sibutramine in animal studies $[11,12]$. 
Thus, three clear messages emerge from the literature. First, the degree of energy expenditure-related weight loss that can be induced pharmacologically in rodents is generally much greater than that seen in humans. Secondly, modest thermogenic effects in man could make a valuable direct or indirect contribution to body weight management by reducing the propensity for weight loss to plateau due to metabolic compensation [13]. Thirdly, poor mechanistic specificity of previous weight loss compounds suggests that energy expenditure should arguably be included in the mechanistic studies of developmental compounds.

From this introduction, it will follow that studying the responses to novel pharmacological interventions requires measurement of changes in body composition, changes in energy expenditure, and changes in food intake. Welldocumented reference compounds are of value in providing positive control.

Demonstrating the efficacy of compounds in earlyphase drug development requires particularly sensitive and precise measurement techniques. We have recently reported the potential for quantitative magnetic resonance (QMR) methodology to enhance the precision of body composition change measurements [14] and have documented its precision in a way which will contribute to the design and powering of our studies of pharmacological agents. Here we present a complementary analysis of the precision with which calorimetric methods may be used to detect changes in energy expenditure and macronutrient oxidation rates. To provide a pharmacological context for this analysis, we have adopted ephedrine, an established compound with documented thermogenic properties, to generate the manipulation of energy expenditure in this study.

Recent observations of active brown adipose tissue (BAT) in man may offer new targets through which energy expenditure contributions to weight loss and its maintenance may be enhanced (refs) and may involve attempts to promote the development of BAT from precursor cells [15].

We anticipate that our findings will inform the design of protocols to evaluate novel therapies in the near future.

\section{Methods}

The study was conducted at Addenbrooke's Centre for Clinical Investigation (ACCI), Addenbrooke's Hospital, Cambridge, UK. Within the ACCI, investigations were undertaken in the GlaxoSmithKline Clinical Unit in Cambridge (CUC) and the Wellcome Trust Clinical Research Facility (WTCRF).

2.1. Participants. Participants were recruited from the GSK CUC panel of healthy volunteers. All were healthy males, with body mass index (BMI, weight/height ${ }^{2}$ ) ranging from 25 to $30 \mathrm{~kg} / \mathrm{m}^{2}$. Participants were screened by review of medical history, physical examination, and laboratory tests. Exclusion criteria included history of metabolic disorders, substantial change, or modification of body weight, regular strenuous exercise, or use of recreational drugs. Fourteen subjects completed this study.
The study was performed in accordance with Good Clinical Practice guidelines and the 1996 version of the Declaration of Helsinki. Protocols were approved by an internal GSK review panel, Addenbrooke's Hospital R\&D office, the WTCRF Scientific Advisory Board, and the Cambridge Local Research Ethics Committee (LREC04/Q0108/6). Each subject gave written informed consent prior to participation.

2.2. Treatment. The study followed a double-blind, placebo controlled design. The active treatment was ephedrine hydrochloride $15 \mathrm{mg}$ administered four times per day (E). Eight participants received ephedrine and six received placebo in a randomized order.

2.3. Study Design. Participants attended on two occasions separated by 2 weeks. The days of the study visits are identified as follows: Day -1, Day 1, Day 2, Day 14, and Day 15. Two participants were studied at each visit. Alcohol, caffeine, and strenuous exercise were prohibited 48 hours prior to the first visit and throughout the study.

Participants arrived fasting at 08:00 on Day -1 . Breakfast was given at 09:45 and lunch at 13:30. Following lunch, body composition was measured by dual-energy X-ray absorptiometry (DXA; GE Lunar Prodigy, GE Healthcare, Madison, WI, USA) and by BOD POD (Life Measurement Inc., Concord, CA). Dinner was served at 19:00. After dinner, participants were fitted with an ActiHeart combined activity and heart rate monitor (CamNtech Ltd., Cambridge, UK). The ActiHeart monitors were worn until the end of the visit. At 20:00, each participant entered a room calorimeter, where he remained until 09:00 on Day 2.

Participants followed a strictly defined activity protocol within the calorimeter. During the first evening (Day -1), participants remained sedentary. They prepared for bed at 23:00, and "lights out" was at 23:30. At 07:00 (Day 1), they were woken for blood pressure measurements, to pass urine and to provide fasting blood samples. They returned to bed until 08:00 when basal metabolic rate (BMR) was measured over 1h. At 9:00, they rose and dressed, and breakfast was served at 9:30. To obtain postprandial measures of energy expenditure (not reported here), participants remained recumbent on the bed between 10:00 and 13:00, but they were free to read or watch television. Lunch was served at 13:30. After lunch, participants undertook sedentary activities until a period of cycling exercise at 17:00. During each exercise period, a work rate of 50 watts was maintained for 30 minutes, equivalent to $3 \mathrm{~kJ} / \mathrm{min}$ external work, costing to the subject typically $13 \mathrm{~kJ} / \mathrm{min}$. Dinner was served at 19:00. Following this, the first treatment dose was given at 20:00. A further period of exercise began at 21:00. Participants prepared for bed at 23:00 as on the previous night. The protocol described above was then repeated until participants left the calorimeter at 22:00 following the exercise on the evening of Day 2. During Day 2, ephedrine or placebo was administered at 07:00, 13:00, 16:00, and 20:00. At each treatment time point, blood pressure was recorded and a blood sample was drawn. Treatment administration continued until the end of the second visit. 
During the second visit, Day 14 events were as those for Day -1 with the exception that body composition measurements (DXA and BOD POD) were not repeated. Day 15 events were as those for Day 2.

2.4. Calorimetry. The room calorimeters, in the WTCRF, are comfortable bed-sitting rooms. They provide measures of oxygen consumption and carbon dioxide production. Urine passed by the subject is collected and analysed for urea and creatinine, from which nitrogen excretion is calculated. From these measures, macronutrient oxidation rates (fat, $\mathrm{CHO}$, protein) are computed and energy expenditure is derived. Calculations, calibrations, and performance have been described in previous publications [16-18].

The rooms are approximately $4 \mathrm{~m} \times 2.5 \mathrm{~m}$ in area and $2.15 \mathrm{~m}$ high and have a window to the outside world. Two air-lock hatches allow entry of food and exit of urine samples. A porthole, sealed with a latex sleeve, enables a participant to present an arm for venepuncture. The rooms are carpeted and are furnished with a bed, table, armchair, and desk chair. They have a wash-basin and a caravan-type flushable chemical toilet. A television with DVD and games console and a computer with internet access are provided for recreation. Exercise is performed on a Seca Cardiotest CT100 cycle ergometer (Seca GMBH, Hamburg, Germany).

2.5. Diet. Prior to attendance for the study, each participant's daily energy expenditure within the calorimeter was predicted using an estimate of BMR from the regression equations of Schofield [19] scaled to account for sedentary activity, and with an addition for the cost of programmed exercise. Diets were prescribed to match these individual predictions. Diets were presented as three meals of equal energy content and composition, with $35 \%$ of energy as fat, $13 \%$ as protein, and $52 \%$ as carbohydrate. Decaffeinated drinks were provided ad libitum, but the associated energy was fixed by the provision of a standardised milk allowance and low-energy sweeteners.

2.6. Statistical Analysis. Ephedrine was compared with placebo by comparing the difference in active treatment measurements between days or visits with the corresponding difference in placebo treatment measurements. Analysis was performed using the general linear model implemented in the DataDesk 6.0 statistical analysis application (Data Description Inc., Ithaca, NY, USA). The significance level, $\alpha$, was set at 0.05 .

\section{Results}

The physical characteristics of the participants are presented in Table 1. Mean values were quite well matched between the ephedrine and placebo participants, with no significant difference between groups in any measured characteristic.

Variability in repeated measures of energy expenditure and macronutrient oxidation was explored in the placebo group. Two repeat intervals were explored: successive days and two days separated by 14 days. Analysis was performed

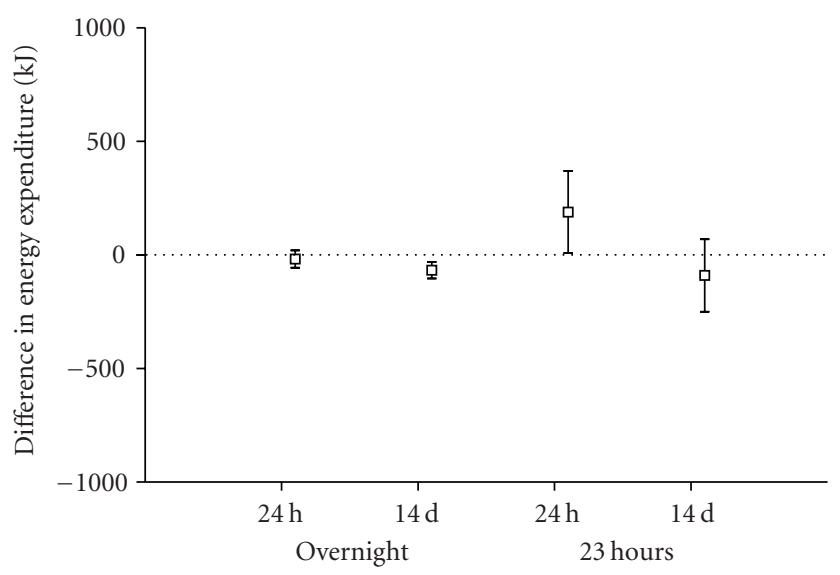

Figure 1: Mean differences and standard deviations of differences between measures of energy expenditure, made overnight and over 23 hours, and repeated after a 24-hour or 14-day interval. Untreated (placebo) subjects, $n=6$.

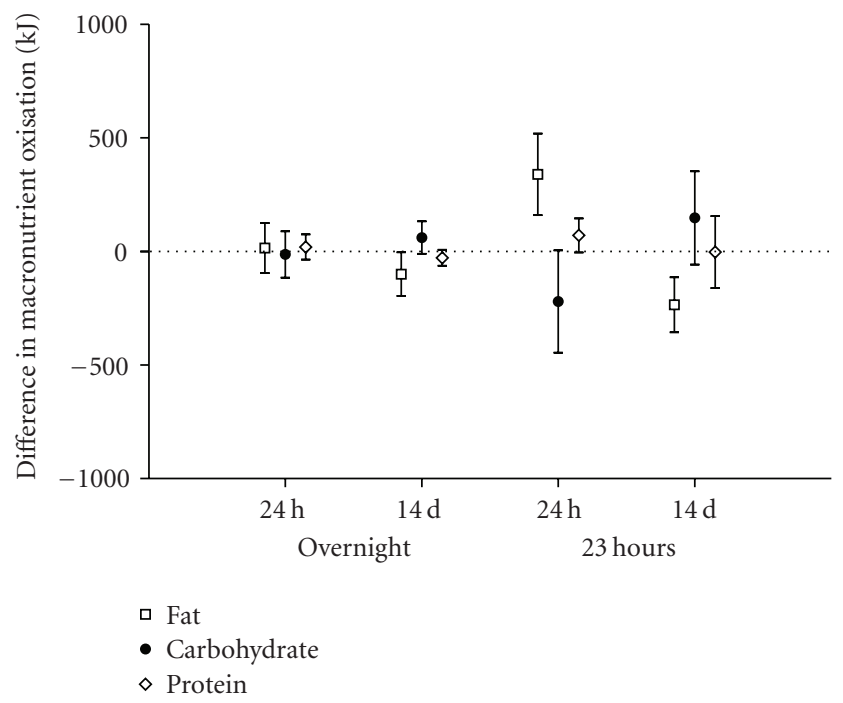

Figure 2: Mean differences and standard deviations of differences between measures of fat, carbohydrate, and protein oxidation, made overnight and over 23 hours, and repeated after a 24-hour or 14-day interval. Untreated (placebo) subjects, $n=6$.

between 00:00 and 07:00 representing sleep, and over a 23hour interval between 22:00 and 21:00 the following day. Due to a technical problem during the data acquisition on day 14, data for one placebo subject was excluded from the 23-hour variability analyses and from the treatment effect analysis. Figures 1 and 2 illustrate the findings of the variability analysis. From this analysis, we estimate that the methodology has the power to detect the changes in energy expenditure and macronutrient oxidation presented in Tables 2(a) and 2(b).

Figure 3 illustrates the concentrations of ephedrine hydrochloride in the circulation of the actively treated participants. Though the plasma concentration during the night following the initial dose was lower than on the 
TABLE 1: Physical characteristics of ephedrine study subjects.

\begin{tabular}{lcccc}
\hline Study & \multicolumn{2}{c}{ Treated: $n=8$} & \multicolumn{2}{c}{ Placebo $n=6$} \\
\hline Age $(\mathrm{y})$ & Mean & SD & Mean & 30.60 \\
Height $(\mathrm{m})$ & 29.42 & 3.69 & 1.75 & 0.03 \\
Weight $(\mathrm{kg})$ & 1.81 & 0.07 & 81.00 & 3.62 \\
BMI $\left(\mathrm{kg} / \mathrm{m}^{2}\right)$ & 86.76 & 5.01 & 26.49 & 1.24 \\
Fat-free mass $(\mathrm{kg})$ & 26.57 & 1.48 & 62.61 & 3.30 \\
Fat mass $(\mathrm{kg})$ & 63.70 & 7.32 & 18.39 & 5.71 \\
Fat $(\%)$ & 23.05 & 4.04 & 22.52 & 6.15 \\
\hline
\end{tabular}

TABLE 2

(a) The predicted change in energy expenditure detectable in response to an intervention in a group of 6 subjects.

\begin{tabular}{lcc}
\hline & Consecutive days change in EE kJ & 14-day-apart change in EE kJ \\
\hline Overnight $(7 \mathrm{~h})$ & 40 & 38 \\
$24 \mathrm{~h}$ & 190 & 202 \\
\hline
\end{tabular}

(b) The predicted change in macronutrient oxidation detectable in a group of 6 subjects.

\begin{tabular}{lcccccc}
\hline & \multicolumn{3}{c}{ Consecutive days change in kJ } & \multicolumn{3}{c}{ 14-day-apart change in kJ } \\
& Protein & CHO & Fat & Protein & CHO & Fat \\
\hline Overnight $(7 \mathrm{~h})$ & 58 & 107 & 116 & 37 & 76 & 101 \\
$24 \mathrm{~h}$ & 79 & 237 & 189 & 163 & 212 & 125 \\
\hline
\end{tabular}

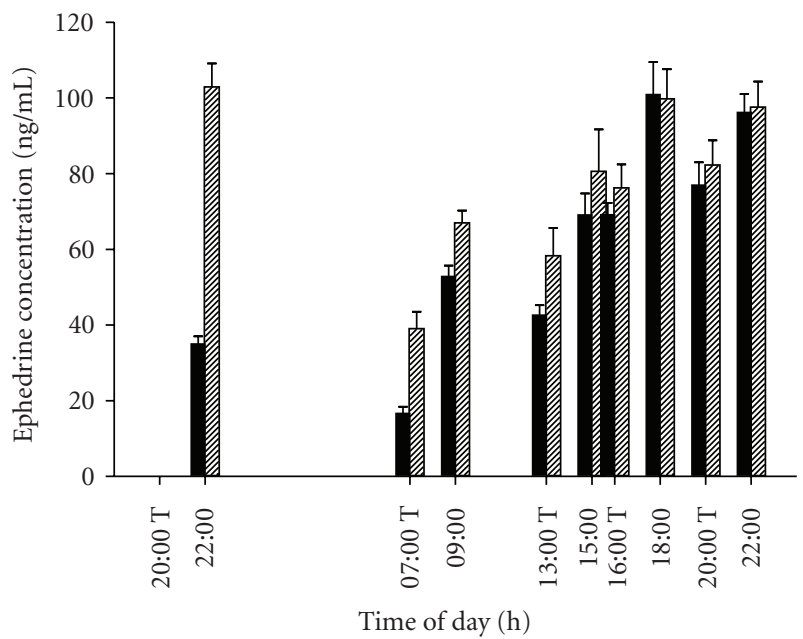

FIGURE 3: Circulating concentrations of ephedrine hydrochloride in treated subjects $(n=8)$. Solid bars: days 1-2; hatched bars days: 1415. $\mathrm{T}$ indicates administration times.

corresponding night of the second visit, the daytime concentrations were similar on both visits.

Figure 4 shows the influence of ephedrine treatment on heart rate, measured over 22.5 hours, between 23:00 and 21:30 the next day. Treatment significantly increased heart rate both acutely ( 5.5 beats $/ \mathrm{min}$ ) and chronically (3.8 beats $/ \mathrm{min}$ ) when compared to the placebo treated group.

Table 3 presents the average macronutrient oxidation and energy expenditure for all subjects, overnight and over 23 hours, on the untreated day (Day -1$)$. This provides

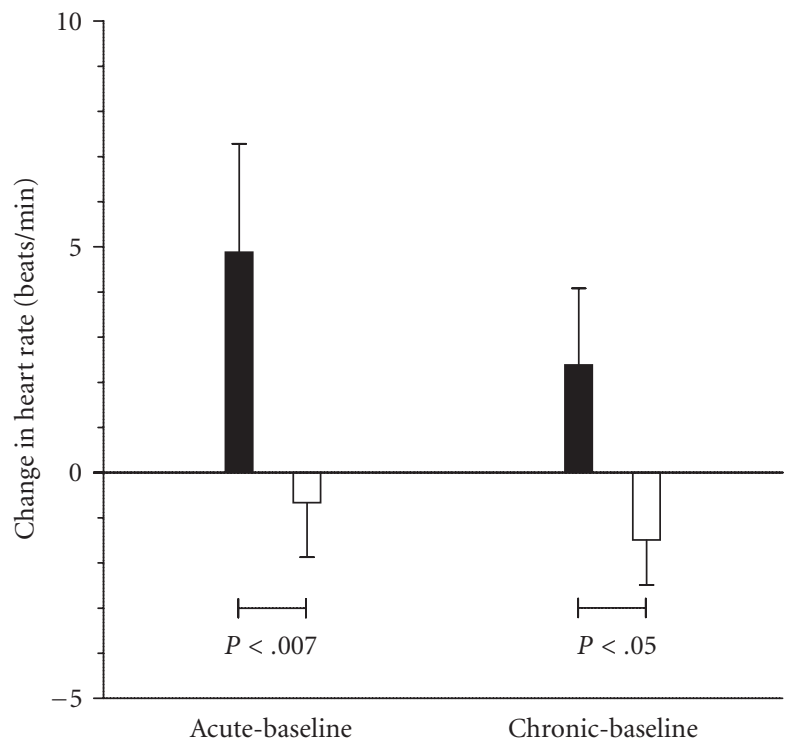

Figure 4: Influence on 22.5-hour average heart rate of acute or chronic (14 day) treatment with ephedrine hydrochloride (solid bars) or placebo (open bars).

a context for interpreting the magnitude of the treatment effects, which are presented in Table 4.

The differences in overnight energy expenditure and macronutrient oxidation between ephedrine- and placebotreated participants are presented in Table 4(a). Ephedrine elevated energy expenditure significantly relative to placebo between both the acute- and chronic-treated nights and 
TABLE 3: Macronutrient oxidation and energy expenditure-means for all subjects in the untreated condition.

\begin{tabular}{|c|c|c|c|c|c|c|c|c|}
\hline & \multicolumn{2}{|c|}{ Protein $(\mathrm{kJ})$} & \multicolumn{2}{|c|}{$\mathrm{CHO}(\mathrm{kJ})$} & \multicolumn{2}{|c|}{ Fat (kJ) } & \multicolumn{2}{|c|}{ Energy $(\mathrm{kJ})$} \\
\hline & Mean & $\mathrm{SD}$ & Mean & $\mathrm{SD}$ & Mean & SD & Mean & SD \\
\hline Overnight (7h) & 358 & 154 & 684 & 220 & 1033 & 253 & 2075 & 145 \\
\hline $23 \mathrm{~h}$ & 1356 & 240 & 4463 & 680 & 4137 & 938 & 9957 & 942 \\
\hline
\end{tabular}

TABLE 4

(a) Differences between ephedrine- and placebo-related changes in overnight macronutrient oxidation and energy expenditure, between treated (days 1 and 14) and untreated (day 0), and between chronic and acute treatments (days 14 and 1)

\begin{tabular}{lccccccc}
\hline $\begin{array}{l}\text { Interval } \\
\text { days }\end{array}$ & $\begin{array}{c}\text { Protein } \\
\text { difference }(\mathrm{kJ})\end{array}$ & $P$ & difference $(\mathrm{kJ})$ & $P$ & difference $(\mathrm{kJ})$ & $P$ & $\begin{array}{c}\text { Eat } \\
\text { difference }(\mathrm{kJ})\end{array}$ \\
\hline $1-0$ & -46.7 & .75 & -41.4 & .86 & 150.8 & .14 & 62.7 \\
$14-0$ & -95.9 & .32 & 10.8 & .98 & 190.3 & .05 & .05 \\
$14-1$ & -46.2 & .73 & 52.2 & .78 & 39.5 & .86 & .001 \\
\hline
\end{tabular}

(b) Differences between ephedrine and placebo treatment effects on changes in macronutrient oxidation and energy expenditure over $23 \mathrm{~h}$, from 22:00 to 21:00, between treated (days 1 and 14) and untreated (day 0), and between chronic and acute treatments (days 14 and 1)

\begin{tabular}{|c|c|c|c|c|c|c|c|c|}
\hline \multirow{2}{*}{$\begin{array}{l}\text { Interval } \\
\text { days }\end{array}$} & \multicolumn{2}{|c|}{ Protein } & \multicolumn{2}{|c|}{ Carbohydrate } & \multicolumn{2}{|l|}{ Fat } & \multicolumn{2}{|l|}{ Energy } \\
\hline & difference $(\mathrm{kJ})$ & $P$ & difference $(\mathrm{kJ})$ & $P$ & difference $(\mathrm{kJ})$ & $P$ & difference $(\mathrm{kJ})$ & $P$ \\
\hline $1-0$ & 131.9 & .52 & 14.5 & .99 & 36.7 & .99 & 183.2 & .48 \\
\hline $14-0$ & 285.8 & .06 & -61.0 & .93 & -94.9 & .92 & 129.9 & .69 \\
\hline $14-1$ & 153.8 & .41 & -75.6 & .90 & -161.6 & .85 & -53.3 & .94 \\
\hline
\end{tabular}

the untreated night, but there was no significant difference between acute and chronic nights. The magnitude of the treatment effect was $3.0 \%$ of the group average untreated overnight energy expenditure (shown in Table 3) for the acute response and $5.1 \%$ for the chronic response. The energy expenditure increments were associated with increments in fat oxidation which reached significance when chronic treatment was compared to the untreated state. There were no significant effects of treatment on carbohydrate or protein oxidation.

Differences in 23-hour energy expenditure and macronutrient oxidation between ephedrine- and placebo-treated participants are shown in Table 4(b). No treatment effects reached significance, though the changes in energy expenditure reflected those which were significant in the overnight observations and represented $1.8 \%$ and $1.3 \%$ for acute and chronic treatment effects, respectively.

\section{Discussion}

Weight loss agents may act on both sides of the energy balance equation, through central anorexigenic effects or modulation of energy expenditure. The recent resurgence of interest in BAT as a potential mediator of thermogenesis in adult humans [15, 20-23] has refocussed interest on thermogenic targets for potential antiobesity drugs. Increased thermogenesis might be achieved by targeting BAT with small molecules or possibly by increasing the number of brown adipocytes and their related oxidative capacity [24] via either transdifferentiation of white adipocytes [25] and/or shifting the developmental fate of adipocyte progenitors [26].
The evaluation of potential thermogenic compounds requires measures of changes, energy expenditure, macronutrient oxidation, and body composition. Modest rates of body mass reduction yield beneficial clinical outcomes [27], and so high measurement precision is essential to identify compounds with favourable profiles during early stages of development. We have recently reported on the favourable precision of QMR for elucidating changes in body composition [14]. Now, we have explored the changes in energy expenditure and macronutrient oxidation induced by ephedrine administered both acutely and over a 2-week "chronic" interval, an interval over which we might expect to be able to detect the beneficial body composition changes using QMR. We have explored ephedrine as a potential comparator compound and have documented the sensitivity with which whole-body indirect calorimetry can detect modest changes in energy expenditure and macronutrient oxidation following its administration.

Ephedrine has been shown to have a complex mechanism of action which includes central sympathetic activation, direct actions on adrenoreceptors [28], and peripheral noradrenalin release [29]. Both alone [30] and in combination with caffeine [4], it has been shown to increase thermogenesis in man, with much of this activity contributed by skeletal muscle in addition to BAT [31]. We selected ephedrine as our test compound in the light of this literature and, in doing so have extended the literature on what may well become a valuable "positive control" against which new compounds are evaluated.

Our study extends the surprisingly limited literature documenting the effects of ephedrine on energy expenditure 
over the day [30]. Shannon et al. administered ephedrine $50 \mathrm{mg}$ three times per day over 24 hours. This was a rather higher dose than the $15 \mathrm{mg} \times 4$ per day which we administered and which we chose to minimise side effects during the longer period (14 days versus 1 day) of administration necessary to study ephedrine's chronic effects. We found a 3\% increase in overnight energy expenditure after acute administration, rising to $5.1 \%$ after 15 days. This compares with $8.4 \%$ acute response reported by Shannon et al. at their higher dose. When we analysed the 23-hour data (Table 4(b)), we found our effects reduced below the level of significance, in both the acute or chronic conditions, to 1.8 and $1.3 \%$. However, Shannon et al. also reported attenuated 24-hour effects, to a just-significant 3.6\%. Given the robust response in the overnight measurements, this suggests a substantial compensation in daytime energy expenditure for the effects of the ephedrine treatment, at least at the thermoneutral temperatures of our studies.

Our study extends the report of Shannon et al. by demonstrating that the increment in overnight energy expenditure was funded by increased fat oxidation, significantly so over the chronic study period. This supports the inference from early, short-duration resting gas exchange measurements made on directly-expired air [32]. The recruitment of fat oxidation seen overnight was not present in the 23 hours analysis.

The effect of ephedrine on heart rate was significant and was maintained to the end of the 14-day intervention (Figure 4), suggesting little tachyphylaxis of its cardiac chronotropic effects during chronic ephedrine administration. The magnitude of the rise in heart rate is similar to that seen with sibutramine treatment [33]. The lack of tachyphylaxis is confirmed by the overnight energy expenditure response to ephedrine. Compared to the placebotreated group, the energy expenditure of the ephedrine group was significantly elevated during the overnight period (Table 4(a)). During the first night, after a single ephedrine dose (Figure 3), the elevation was 3\%. After 14 days, this had increased to $5 \%(P<.001)$, by which time circulating ephedrine levels (at steady state) were quantifiable 2 hours post dose (Figure 3).

Data from the control group in our study extends the literature documenting the intraindividual variability of 24hour or overnight energy expenditure and macronutrient oxidation rates, both from day-to-day and across longertime intervals [34-36]. Though such information is essential to the design and powering of studies seeking to document the effects of interventions which target energy expenditure or macronutrient balance, we found insufficient published work to enable us to power studies with confidence. Murgatroyd et al. [37] reported day-to-day variability in energy expenditure over 14-day continuous confinement in a calorimeter. Schoffelen and Westerterp [35] focussed on variability in overnight observations. Rumpler et al. [36] looked at short- and longer-term repeatability, as we have done here, but the longer-term analysis may have been influenced by energy imbalance. None of these reports extend to analysis of variability in macronutrient oxidation rates.
Taking data from the placebo-treated group, we have assessed the repeatability of these measurements and have derived the sensitivity with which putative changes in energy expenditure and macronutrient oxidation might be resolved in an overnight resting state or over a whole day. In the context of the expenditure and oxidation levels in Table 3, we estimate that treatment-induced changes in energy expenditure greater than $2 \%$ should be detectable in a group of six subjects (Table $2(\mathrm{a})$ ), while changes in fat or carbohydrate oxidation should be detectable at around the 5\% level (Table 2(b)). This suggests that calorimetry offers sufficient sensitivity to detect quite subtle changes in energy expenditure and fuel selection in response to pharmacological interventions, changes which nonetheless could have valuable therapeutic benefits in the medium-tolong term.

\section{Conclusions}

In conclusion, highly precise methods are paramount for detecting subtle differences in energy expenditure when evaluating the thermogenic properties of drugs that are being evaluated for the treatment of obesity. Our data confirms that modest drug effects, where present, can be detected by whole-body indirect calorimetry, and this technique should be considered when planning small experimental medicine studies to evaluate manipulations of energy balance. The high degree of precision in detecting changes in both energy expenditure and macronutrient utilisation should permit early confirmation of preclinical data and proof of mechanism and help to predict long-term efficacy. We have confirmed that ephedrine is a reliable thermogenic compound against which comparison of novel thermogenic agents may be made after both acute and chronic administration.

\section{Acknowledgments}

The authors thank the following for their contributions to this study: Allison Brooke and Eilis O'Driscol for GSK CUC their operational and technical support throughout the study, Elaine Marriott and Samantha Northcott WTCRF for coordinating the study in the WTCRF, and all of the subjects who participated in this study. Calorimetric investigations are supported through the MRC Centre for Obesity and Related Metabolic Disorders (MRC-CORD). Dr. Nick Finer is a recipient of an unrestricted grant from GlaxoSmithKline. This study was sponsored by GlaxoSmithKline.

\section{References}

[1] T. A. Wadden, R. I. Berkowitz, L. G. Womble et al., "Randomized trial of lifestyle modification and pharmacotherapy for obesity," The New England Journal of Medicine, vol. 353, no. 20, pp. 2111-2120, 2005

[2] A. Avenell, J. Broom, T. J. Brown et al., "Systematic review of the long-term effects and economic consequences of treatments for obesity and implications for health improvement," Health Technology Assessment, vol. 8, no. 21, pp. 1-182, 2004. 
[3] M. L. Dansinger, A. Tatsioni, J. B. Wong, M. Chung, and E. M. Balk, "Meta-analysis: the effect of dietary counseling for weight loss," Annals of Internal Medicine, vol. 147, no. 1, pp. 41-50, 2007.

[4] A. Astrup, L. Breum, S. Toubro, P. Hein, and F. Quaade, "The effect and safety of an ephedrine/caffeine compound compared to ephedrine, caffeine and placebo in obese subjects on an energy restricted diet. A double blind trial," International Journal of Obesity and Related Metabolic Disorders, vol. 16, no. 4, pp. 269-277, 1992.

[5] A. Astrup, S. Madsbad, L. Breum, T. J. Jensen, J. P. Kroustrup, and T. M. Larsen, "Effect of tesofensine on bodyweight loss, body composition, and quality of life in obese patients: a randomised, double-blind, placebo-controlled trial," The Lancet, vol. 372, no. 9653, pp. 1906-1913, 2008.

[6] A. Astrup, S. Toubro, N. J. Christensen, and F. Quaade, "Pharmacology of thermogenic drugs," The American Journal of Clinical Nutrition, vol. 55, no. 1, pp. 246S-248S, 1992.

[7] D. L. Hansen, S. Toubro, M. J. Stock, I. A. Macdonald, and A. Astrup, "Thermogenic effects of sibutramine in humans," The American Journal of Clinical Nutrition, vol. 68, no. 6, pp. 1180 1186, 1998.

[8] D. L. Hansen, S. Toubro, M. J. Stock, I. A. Macdonald, and A. Astrup, "The effect of sibutramine on energy expenditure and appetite during chronic treatment without dietary restriction," International Journal of Obesity and Related Metabolic Disorders, vol. 23, no. 10, pp. 1016-1024, 1999.

[9] H. M. Seagle, D. H. Bessesen, and J. O. Hill, "Effects of sibutramine on resting metabolic rate and weight loss in overweight women," Obesity Research, vol. 6, no. 2, pp. 115121, 1998.

[10] R. D. Starling, X. Liu, and D. H. Sullivan, "Influence of sibutramine on energy expenditure in African American women," Obesity Research, vol. 9, no. 4, pp. 251-256, 2001.

[11] I. P. Connoley, Y.-L. Liu, I. Frost, I. P. Reckless, D. J. Heal, and M. J. Stock, "Thermogenic effects of sibutramine and its metabolites," British Journal of Pharmacology, vol. 126, no. 6, pp. 1487-1495, 1999.

[12] I. P. Connoley, Y.-L. Liu, I. Frost, I. P. Reckless, D. J. Heal, and M. J. Stock, "Thermogenic effects of sibutramine and its metabolites," British Journal of Pharmacology, vol. 126, no. 6, pp. 1487-1495, 1999.

[13] M. Rosenbaum, J. Hirsch, D. A. Gallagher, and R. L. Leibel, "Long-term persistence of adaptive thermogenesis in subjects who have maintained a reduced body weight," The American Journal of Clinical Nutrition, vol. 88, no. 4, pp. 906-912, 2008.

[14] A. Napolitano, S. R. Miller, P. R. Murgatroyd et al., "Validation of a quantitative magnetic resonance method for measuring human body composition," Obesity, vol. 16, no. 1, pp. 191198, 2008.

[15] J. Nedergaard, T. Bengtsson, and B. Cannon, "Unexpected evidence for active brown adipose tissue in adult humans," American Journal of Physiology-Endocrinology and Metabolism, vol. 293, no. 2, pp. E444-E452, 2007.

[16] D. Brown, T. J. Cole, and M. J. Dauncey, "Analysis of gaseous exchange in open-circuit indirect calorimetry," Medical and Biological Engineering and Computing, vol. 22, no. 4, pp. 333338, 1984.

[17] M. Elia, G. Livesey, et al., "Energy expenditure and fuel selection in biological systems: the theory and practice of calculations based on indirect calorimetry and tracer methods," in Control of Eating, Energy Expenditure and the Bioenergetics of Obesity, A. P. Simopoulos, Ed., vol. 70 of World Review in
Nutrition and Dietetics, pp. 68-131, Karger, Basel, Switzerland, 1992.

[18] P. R. Murgatroyd, B. J. Sonko, A. Wittekind, G. R. Goldberg, S. M. Ceesay, and A. M. Prentice, "Non-invasive techniques for assessing carbohydrate flux: I. Measurement of depletion by indirect calorimetry," Acta Physiologica Scandinavica, vol. 147, no. 1, pp. 91-98, 1993.

[19] W. N. Schofield, "Predicting basal metabolic rate, new standards and review of previous work," Human Nutrition, vol. 39C, pp. 5-41, 1985.

[20] A. M. Cypess, S. Lehman, G. Williams et al., "Identification and importance of brown adipose tissue in adult humans," The New England Journal of Medicine, vol. 360, no. 15, pp. 15091517, 2009.

[21] M. Saito, Y. Okamatsu-Ogura, M. Matsushita et al., "High incidence of metabolically active brown adipose tissue in healthy adult humans: effects of cold exposure and adiposity," Diabetes, vol. 58, no. 7, pp. 1526-1531, 2009.

[22] W. D. Van Marken Lichtenbelt, J. W. Vanhommerig, N. M. Smulders et al., "Cold-activated brown adipose tissue in healthy men," The New England Journal of Medicine, vol. 360, no. 15, pp. 1500-1508, 2009.

[23] M. C. Zingaretti, F. Crosta, A. Vitali et al., "The presence of UCP1 demonstrates that metabolically active adipose tissue in the neck of adult humans truly represents brown adipose tissue," The FASEB Journal, vol. 23, no. 9, pp. 3113-3120, 2009.

[24] K. Won Park, D. S. Halperin, and P. Tontonoz, "Before they were fat: adipocyte progenitors," Cell Metabolism, vol. 8, no. 6, pp. 454-457, 2008.

[25] S. Cinti, "The role of brown adipose tissue in human obesity," Nutrition, Metabolism and Cardiovascular Diseases, vol. 16, no. 8, pp. 569-574, 2006.

[26] B. M. Spiegelman, P. Seale, and S. Kajimura, "Transcriptional control of brown adipocyte development and physiological function-of mice and men," Genes and Development, vol. 23, no. 7, pp. 788-797, 2009.

[27] K. Brown and S. Twaddle, Management of Obesity: A National Clinical Guideline, Scottish Intercollegiate Guidelines Network, Edinburgh, Scotland, 2010.

[28] B. B. Hoffman and R. J. Lefkowitz, "Catecholamines, sympathomimetic drugs, and adrenergic receptor antagonists," in Goodman and Gilman's The Pharmacological Basis of Therapeutics, J. G. Hardman, L. E. Limbird, P. B. Molinof, R. W. Ruddon, and A. G. Gilman, Eds., pp. 199-248, McGrawHill, New York, NY, USA, 1996.

[29] A. R. Boobis, D. Burley, D. M. Davies, et al., "Ephedrine (hydrochloride)," in Therapeutic Drugs, C. Dollery, Ed., pp. E26-E29, Churchill Livingstone, New York, NY, USA, 1991.

[30] J. R. Shannon, K. Gottesdiener, J. Jordan et al., "Acute effect of ephedrine on 24-h energy balance," Clinical Science, vol. 96, no. 5, pp. 483-491, 1999.

[31] A. Astrup, J. Bülow, J. Madsen, and N. J. Christensen, "Contribution of BAT and skeletal muscle to thermogenesis induced by ephedrine in man," The American Journal of Physiology, vol. 248, no. 5, pp. E507-E515, 1985.

[32] A. Astrup, J. Madsen, J. J. Holst, and N. J. Christensen, “The effect of chronic ephedrine treatment on substrate utilization, the sympathoadrenal activity, and energy expenditure during glucose-induced thermogenesis in man," Metabolism, vol. 35, no. 3, pp. 260-265, 1986.

[33] C. Torp-Pedersen, I. Caterson, W. Coutinho et al., "Cardiovascular responses to weight management and sibutramine in high-risk subjects: an analysis from the SCOUT trial," European Heart Journal, vol. 28, no. 23, pp. 2915-2923, 2007. 
[34] P. R. Murgatroyd, H. L. Davies, and A. M. Prentice, "Intraindividual variability and measurement noise in estimates of energy expenditure by whole body indirect calorimetry," British Journal of Nutrition, vol. 58, no. 3, pp. 347-356, 1987.

[35] P. F. M. Schoffelen and K. R. Westerterp, "Intra-individual variability and adaptation of overnight- and sleeping metabolic rate," Physiology and Behavior, vol. 94, no. 2, pp. 158-163, 2008.

[36] W. V. Rumpler, J. L. Seale, J. M. Conway, and P. W. Moe, "Repeatability of 24-h energy expenditure measurements in humans by indirect calorimetry," The American Journal of Clinical Nutrition, vol. 51, no. 2, pp. 147-152, 1990.

[37] P. R. Murgatroyd, H. L. Davies, and A. M. Prentice, "Intraindividual variability and measurement noise in estimates of energy expenditure by whole body indirect calorimetry," British Journal of Nutrition, vol. 58, no. 3, pp. 347-356, 1987. 


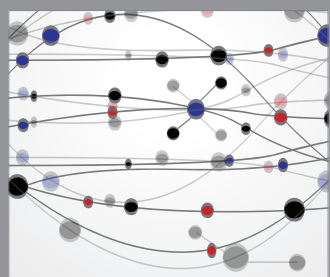

The Scientific World Journal
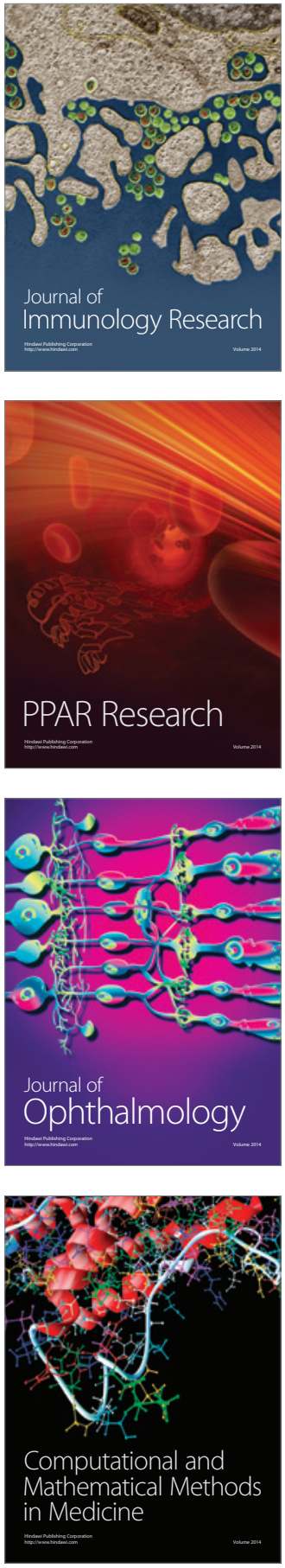

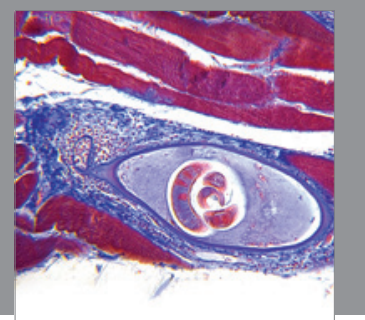

Gastroenterology

Research and Practice
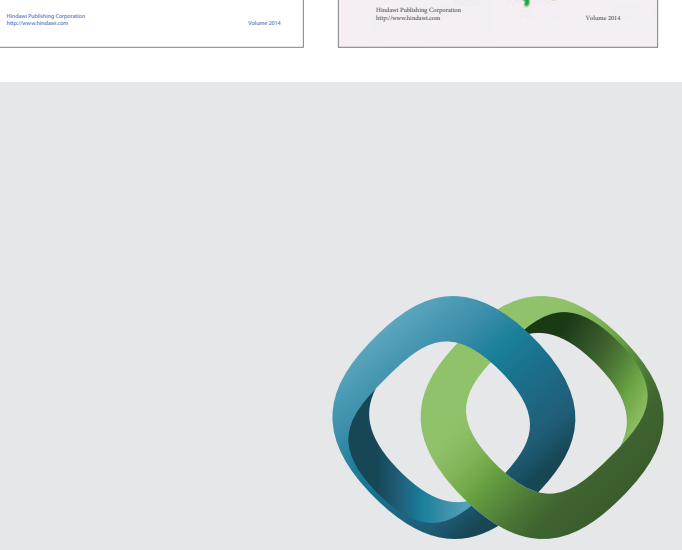

\section{Hindawi}

Submit your manuscripts at

http://www.hindawi.com
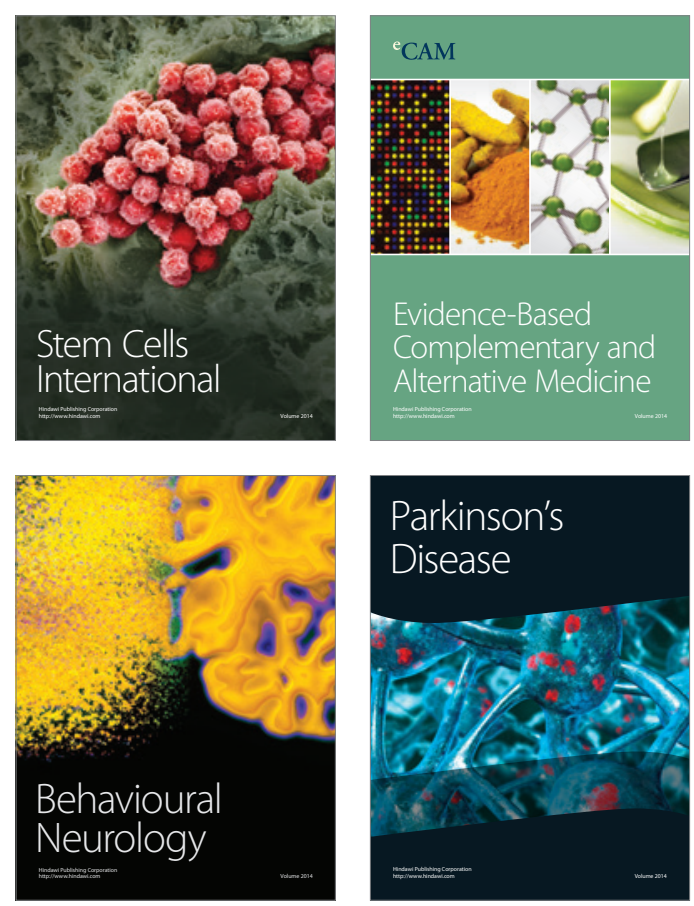

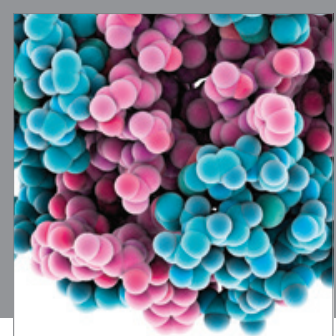

Journal of
Diabetes Research

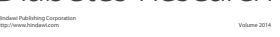

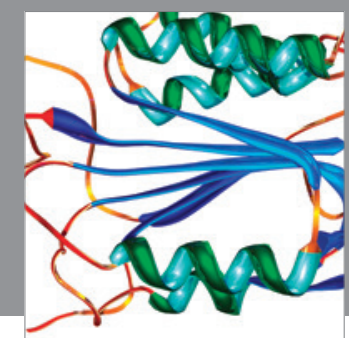

Disease Markers
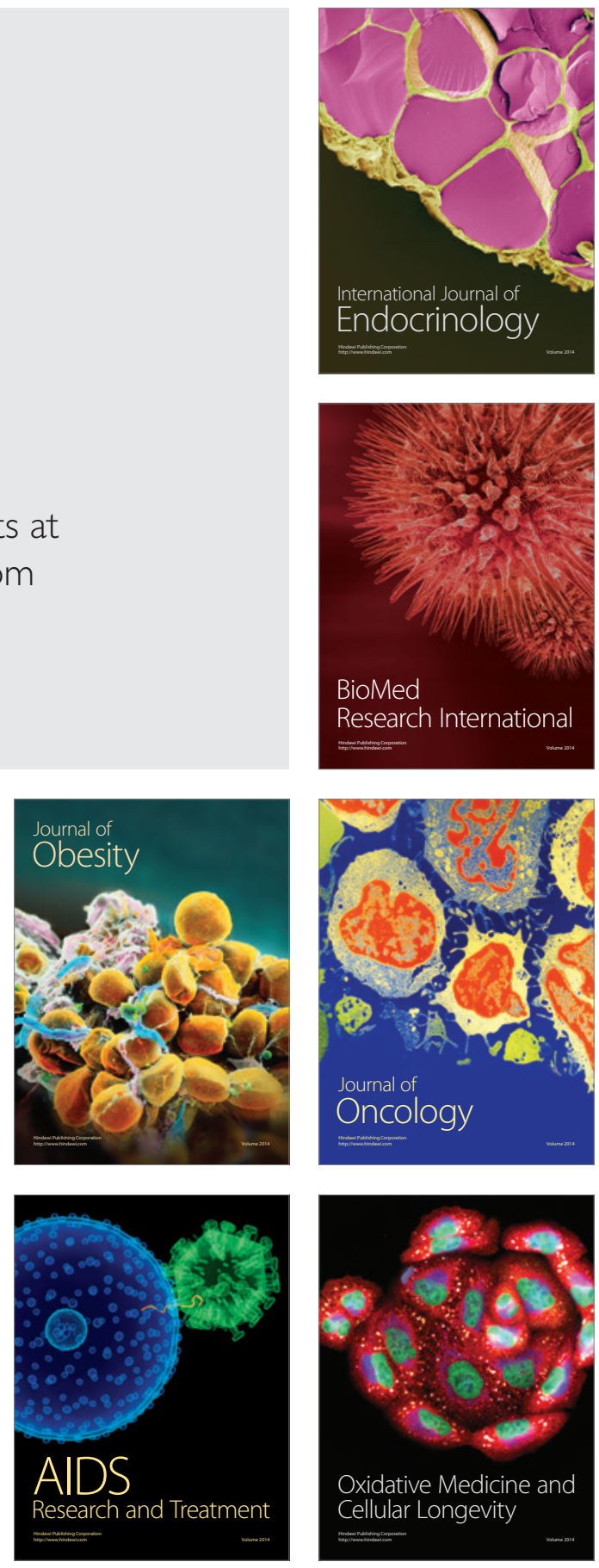